\title{
Caries and dental erosion: are Soroti children and adolescents at risk from increased soft-drink availability in Uganda?
}

\author{
Jordan Cheng ${ }^{1,2}$, Karen Campbell ${ }^{1,2,3}$
}

1. Faculty of Dentistry, University of British Columbia, Canada.

2. Office of Pediatric Surgical Evaluation and Innovation, British Columbia, Canada.

3. Department of Pediatric Dentistry, British Columbia Children's Hospital, Canada.

All authors have made substantive contribution to this study and/or manuscript, and all have reviewed the final paper prior to its submission.

\begin{abstract}
:
Objective: An initial field study to investigate dental caries and dental erosion in children and adolescents in the community of Soroti, Uganda.

Methods: A stratified two-stage cluster sample of 84 children (ages 8-10) and adolescents (ages 16-19) were recruited. A survey was undertaken to assess the state of determinants of oral health, oral hygiene practices, and soft-drink and sweetened-tea consumption. Intra-oral photographs were taken and reviewed to measure Decayed, Missing, and Filled Teeth (DMFT) and dental erosion.
\end{abstract}

Results: A significant difference was observed in DMFT between children and adolescents (3.7 \pm 2.7 versus $2.1 \pm 2.4 \mathrm{p}=.02)$. A trend of lower erosion scores was seen in children compared to adolescents. Greater frequency of sweetened-tea consumption over soft-drinks was noted in both groups.

Conclusion: This study did not reveal any relationship between different levels of cariogenic beverage consumption and DMFT or dental erosion in this sample group.

Keywords: Caries, dental erosion, soft-drink availability in Uganda.

DOI: http://dx.doi.org/10.4314/ahs.v16i4.9

Cite as: Jordan C, Karen C. Caries and dental erosion: are Soroti children and adolescents at risk from increased soft-drink availability in Uganda? Afri Health Sci 2016;16(4): 943-946. bttp:// dx.doi.org/10.4314/abs.v16i4.9

\section{Background}

The lack of nutritional value, high sugar content, and low $\mathrm{pH}$ render soft-drinks a real threat to the developing dentition. ${ }^{1,2,3,4}$ Previously, minimal focus was given to the additional negative effect of dental erosion on the dentition. ${ }^{5,6,7,8,9,10,11}$ Dental erosion is the permanent loss of enamel from repeated acid exposure without the direct involvement of bacteria. ${ }^{3,12}$ It has been shown that frequent soft-drink consumption contributes to dental erosion. $^{13,14}$

A study in Kampala, Uganda showed that $24-37 \%$ of older children (10-14 years) consumed soft-drinks daily. ${ }^{5}$ Previous reports have indicated that school children in

\footnotetext{
Corresponding author:

Jordan Cheng,

Dental Student

Faculty of Dentistry, UBC

2199 Wesbrook Mall

Vancouver BC V6T 1 Z3

Email: jordan.cheng@alumni.ubc.ca
} due to the high cost of dental care, lack of established preventive oral health practices, and delayed treatment due to lack of access to dental professionals. ${ }^{6,15}$ A number of school-based surveys have reported increasing caries prevalence in Ugandan school children(ages 5-22). ${ }^{7,8}$

The objective for this study was to carry out a pilot study to explore the oral health determinants and prevalence of dental caries and erosion in Soroti children and adolescents. We aimed to obtain baseline data to determine feasibility for future studies.

It is hypothesized that Soroti students are at risk for increased dental erosion and poorer long-term oral health outcomes from increased soft-drink and or sweetened tea consumption.

\section{Methods}

A stratified two stage cluster sampling method was used to recruit a total of 84 children and adolescents from both public and private schools in Soroti, Uganda. The study included two parts: for each subject, a pre-tested survey

African Health Sciences Vol 16 Issue 4, December, 2016 
and a standardized series of intra-oral photographs of the dentition were obtained. The survey provided information on socio-economic status, details of dental care access, oral hygiene practices, and weekly consumption of soft-drinks and sweetened-tea.

Responses regarding the consumption of cariogenic beverages (soft-drinks/sweetened tea) were graded as follows; a weekly consumption of $0-5$ beverages as 'low', 6-10 beverages as 'medium' and greater than 10 beverages as 'high'.

Each subject was assigned these categories for soft-drink, sweetened-tea, and for total consumption (sweetened tea and soft-drink combined). Intra-oral photographs were taken to determine the status dentition of the individuals' dentition (Figure 1). The intra-oral photographs were assembled, de-identified and reviewed by panel of three blinded calibrated University of British Columbia Pediatric Dentistry Fellows. DMFT and Erosion Index Scores were determined evaluating each sextant of the arches and these were assigned a score by panel consensus.

\section{Ethical approval}

This study has received Human Ethical Research approval by the UBC-Children's and Women's Clinical Research Ethics Review Board in 2014. Informed consent was obtained from all participating adult subjects, and parents or legal guardians of minors.

\section{Results}

A total of 84 students were recruited: 48 children (mean age $9.1 \mathrm{yr} \pm 0.8$ ) and 36 adolescents (mean age $17.3 \mathrm{yr} \pm$ 1.4) during the month of June, 2014. Five public (72\%), two private $(28 \%)$, and two rural $(28 \%)$ schools were sampled. Roughly half of the sample, $48 \%(n=23)$ of children, and $53 \%(n=19)$ of adolescents were in the "below average" socio-economic group.

In children and adolescent groups, $67 \%(n=32)$ and $47 \%(n=17)$ reported experiencing tooth pain. For both groups the majority $(75 \%(\mathrm{n}=36)$ of children and $61 \%(n=22)$ of adolescents) sought care for dental pain from someone other than a dentist. Seventy-three percent of children ( $n=35)$ reported being unhappy with the appearance of their teeth, mainly due to "decay" or "discolouration". In contrast, $64 \%(n=23)$ of the adolescents indicated satisfaction with their dental appearance.

The children had a DMFT of $3.7 \pm 2.7$ while the adolescents had a DMFT of $(2.1 \pm 2.4)$. The difference was significant with a $\mathrm{p}$ value of $<0.05$. Children $(2.8 \pm 2.1)$ appeared to have a lower Erosion Index score compared to the adolescents $(3.6 \pm 2.3)$ but this was not statistically significant.

The majority $(77 \%(n=65))$ of the total sample utilized a toothbrush and toothpaste while $19 \%(\mathrm{n}=16)$ reported chewing stick use. Interestingly, none of the subjects knew what dental floss was, nor were they aware of the concept of flossing as an adjunct to oral hygiene.

In both groups, a majority reported soft-drink consumption in the "low" category. However, in the same groups, $52 \%(\mathrm{n}=25)$ children reported "medium" to "high" consumers of sweetened-tea.

Adolescents were similarly "low" consumers of softdrinks but their sweetened-tea consumption frequency was lower. Subjects reported that an average of 2 to 3 teaspoons of sugar was added to sweeten their tea. There did not appear to be a statistically significant relationship between the level of consumption of these beverages and DMFT or Erosion Index scores for both groups based on the Kruskal-Wallis Test.

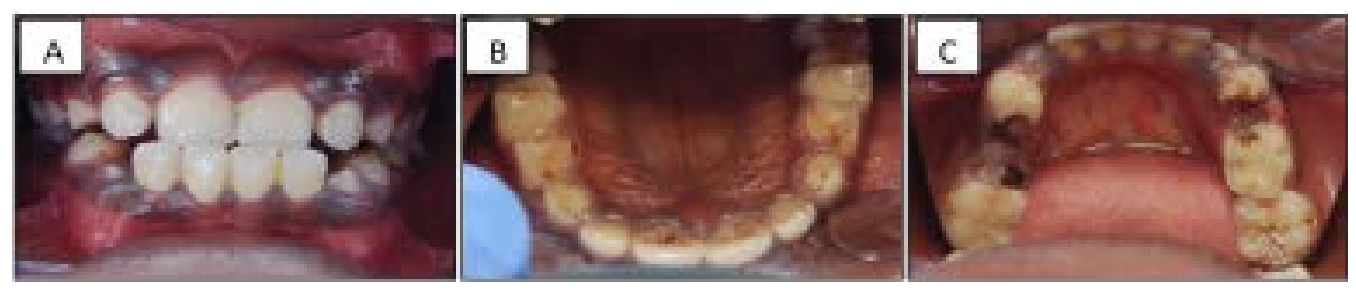

Figure 1. Example of frontal (A), maxillary (B), and mandibular (C) images. All photographs were taken in field conditions.

\section{Discussion}

Limited access to dental services was reported by the participants in our study. Consistent with previous studies, the most common dental complaint was decay. ${ }^{8,10,11,15} \mathrm{It}$ was reported that $80 \%$ of dental surgeons are concentrated in larger urban areas. ${ }^{16}$ As a result, the smaller dis-

African Health Sciences Vol 16 Issue 4, December, 2016 tricts primarily rely on specially trained dental assistants, yet some rural areas may only have traditional healers available. ${ }^{16}$ Since 2007, the Ministry of Health of Uganda has implemented an Oral Health Policy. ${ }^{17}$ Its objectives include providing oral health guidelines, health promotion, training, and framework for evaluation. ${ }^{17}$ 
The majority of subjects reported the use of a toothbrush for dental cleaning. The use of dental floss was not reported in this population. Previous studies on rural and urban Ugandan children do not report dental floss usage. This would be a serious concern as previous studies revealed periodontal disease in their subjects. ${ }^{18,19,20}$

Compared to previous reports on urban Ugandan children, the DMFT scores of our rural child groups were higher $(3.7 \pm 2.7$ vs $1.5 \pm 0.8) .{ }^{15}$ Additionally, these scores were significantly higher in children compared to adolescents $(3.7 \pm 2.7$ vs $2.1 \pm 2.4)$. This contrasted previous studies of other urban and peri-urban locales in Uganda that described an increase in DMFT with age. ${ }^{18,20}$ It has been reported that urban adolescents have higher sugar consumption. ${ }^{5,6}$ In fact, previous studies reported Kampala and Lira adolescents had DMFT scores of 2.4 and 3.3 respectively. ${ }^{6}$ Since the majority of our adolescents are overall less affluent than their urban counterparts, it may account for the lower DMFT.

Uganda is the third-leading producer and exporter of tea in Africa, with annual consumption approximating 3,000 tonnes. ${ }^{21}$ It is likely that for families, sweetened-tea may still be a more affordable and palatable option to softdrinks. The preference for sweetened-tea may account for the low soft-drink consumption level despite increased marketing efforts by major soft-drink companies. Nevertheless, it may be noted that soft-drinks were readily available in Soroti's local markets.

\section{Limitations}

The limitations of this study include; small sample size and inaccuracies from scoring DMFT and Erosion Index from photographs. Furthermore, the sample may not be a representative of the region. The Erosion Index was scored using the photographs and dependence on the quality of the images. Erosion may have been incorrectly interpreted as other forms of wear, such as abrasion and attrition if subtle may not have been detectable. Despite our determination that soft-drink consumption has yet to be a significant contributing factor to poor oral health outcomes in Soroti children and adolescents, it remains crucial for public health practitioners to counsel families on the lack of nutritional value and detrimental effects of acidic sweetened beverages, including sweetened-tea and soft-drinks. With commercial development in the region, a high potential for increased consumption of tea and soft-drinks in this population remains. There is opportu nity to continue advocating for access to dental care and promoting awareness of flossing as part of proper oral hygiene.

\section{Competing interests}

None declared.

\section{Ethical approval}

This study has received Human Ethical Research approval by the UBC-Children's and Women's Clinical Research Ethics Review Board in 2014.

\section{Acknowledgments}

We would like to recognize the Soroti Regional Referral Hospital for their support and partnership for this project. We would also like to thank UBC pediatric dentistry fellows Drs. Jennifer Park, Shan Sun, Anu Korada, Kunal Chander, Andrew Wong, and Zina Alkafaji for assisting with the scoring of the photographs. Finally, we would like to acknowledge Dr. Ravindra Shah for his encouragement and guidance towards publication of the manuscript.

\section{Funding/support}

This study was funded by the Child and Family Research Institute, Vancouver, Canada and the Canadian Pacific Railway, Canada.

\section{References}

1. Harnack L, Stang J, Story M. Soft drink consumption among US children and adolescents: nutritional consequences. J Am Diet Assoc. 1999 Apr;99(4):436-41.

2. Cheng R, Yang H, Shao M, Hu T, Zhou X. Dental erosion and severe tooth decay related to soft drinks: a case report and literature review. J Zhejiang Univ Sci B. 2009 May;10(5):395-9.

3. Clark DC, Woo G, Silver JG, Sweet D, Grisdale JC. The influence of frequent ingestion of acids in the diet on treatment for dentin sensitivity. J Can Dent Assoc. 1990 Dec;56(12):1101-3.

4. Touger-Decker R, van Loveren C. Sugars and dental caries. Am J Clin Nutr. 2003 Oct;78(4):881S-892S.

5. Kiwanuka SN, Astrøm AN, Trovik TA. Sugar snack consumption in Ugandan schoolchildren: Validity and reliability of a food frequency questionnaire. Community Dent Oral Epidemiol. 2006 Oct;34(5):372-80.

6. Okullo I, Astrøm AN, Haugejorden O. Social inequalities in oral health and in use of oral health care services among adolescents in Uganda. Int J Paediatr Dent Br Paedodontic Soc Int Assoc Dent Child. 2004 Sep;14(5):326-35.

African Health Sciences Vol 16 Issue 4, December, 2016 
7. Diehnelt DE, Kiyak HA. Socioeconomic factors that affect international caries levels. Community Dent Oral Epidemiol. 2001 Jun;29(3):226-33.

8. Wandera M, Twa-Twa J. Baseline survey of oral health of primary and secondary school pupils in Uganda. Afr Health Sci. 2003 Apr;3(1):19-22.

9. Kiwanuka SN, Astrøm AN, Trovik TA. Dental caries experience and its relationship to social and behavioural factors among 3-5-year-old children in Uganda. Int J Paediatr Dent Br Paedodontic Soc Int Assoc Dent Child. 2004 Sep;14(5):336-46.

10. Nalweyiso N, Busingye J, Whitworth J, Robinson PG. Dental treatment needs of children in a rural subcounty of Uganda. Int J Paediatr Dent Br Paedodontic Soc Int Assoc Dent Child. 2004 Jan;14(1):27-33.

11. Kutesa A, Mwanika A, Wandera M. Pattern of dental caries in Mulago Dental School clinic, Uganda. Afr Health Sci. 2005 Mar;5(1):65-8.

12. Imfeld T. Dental erosion. Definition, classification and links. Eur J Oral Sci. 1996 Apr;104(2 (Pt 2)):151-5.

13. Järvinen VK, Rytömaa II, Heinonen OP. Risk factors in dental erosion. J Dent Res. 1991 Jun;70(6):942-7.

14. Sayegh A, Dini EL, Holt RD, Bedi R. Food and drink consumption, sociodemographic factors and dental car- ies in 4-5-year-old children in Amman, Jordan. Br Dent J. 2002 Jul 13;193(1):37-42.

15. Batwala V, Mulogo E, Arubaku W. Oral health status of school children in Mbarara, Uganda. Afr Health Sci. 2007 Dec;7(4):233-8.

16. Muhirwe LB. Oral health in Uganda: the need for a change in focus. Int Dent J. 2006 Feb;56(1):3-6.

17. Ministry of Health. National Oral Health Policy. 2007. Available from: http://health.go.ug/content/nationaloral-health-policy-0. Accessed 2016-08-23.

18. Jensen K, Kizito EK, Langebaek J, Nyika TA. Dental caries, gingivitis, and oral hygiene among schoolchildren in Kampala, Uganda. Community Dent Oral Epidemiol. 1973;1(2):74-83.

19. Albandar JM, Muranga MB, Rams TE. Prevalence of aggressive periodontitis in school attendees in Uganda. $J$ Clin Periodontol. 2002 Sep;29(9):823-31.

20. Muwazi LM, Rwenyonyi CM, Tirwomwe FJ, Ssali C, Kasangaki A, Nkamba ME, et al. Prevalence of oral diseases/conditions in Uganda. Afr Health Sci. 2005 Sep;5(3):227-33. 21. Kiwanuka B, Ahmed M. Analysis of incentives and disincentives for tea in Uganda. Rome.

21. Food and Agriculture Organization of the United Nations, 2012. Available at: "http://www.fao.org/3/aat593e.pdf". Accessed 2016-02-19. 